\title{
Evaluation of Human P-Glycoprotein (MDR1/ABCB1) ATPase Activity Assay Method by Comparing with in Vitro Transport Measurements: Michaelis-Menten Kinetic Analysis to Estimate the Affinity of P-Glycoprotein to Drugs
}

\author{
Yoshiyuki Shirasaka, ${ }^{* a}$ Yuko Onishi, ${ }^{b}$ Aki Sakurai,${ }^{b}$ Hiroshi Nakagawa, ${ }^{b}$ Toshihisa Ishikawa, ${ }^{b}$ and \\ Shinji YAMASHITA ${ }^{a}$ \\ ${ }^{a}$ Faculty of Pharmaceutical Sciences, Setsunan University; 45-1 Nagaotoge-cho, Hirakata, Osaka 573-0101, Japan: and \\ ${ }^{b}$ Department of Biomolecular Engineering, Graduate School of Bioscience and Biotechnology, Tokyo Institute of \\ Technology; 4259 Nagatsuta-cho, Midori-ku, Yokohama 226-8501, Japan. \\ Received July 30, 2006; accepted September 7, 2006; published online September 12, 2006
}

\begin{abstract}
Human ABC transporter P-glycoprotein (P-gp/ABCB1) encoded by the multidrug resistance (MDR1) gene is recognized as one of the most important factors regulating pharmacokinetics of a number of clinically important drugs because of its function of extruding a wide range of structurally unrelated amphiphilic and hydrophobic drugs from the inside to the outside of cells in an ATP-driven mechanism. In the present study, we have evaluated the high-speed ATPase activity assay method by comparing with in vitro transport assay systems using MDR1-transfected MDR1-MDCK cells. Since substrate drugs were found to interfere with the photometric detection of inorganic phosphate (Pi) that was liberated according to the hydrolysis of ATP to ADP in ATPase activity assay, at first, a method in which the amount of Pi can be calculated correctly. Results demonstrate that the kinetic parameters obtained in ATPase activity assay are not necessarily correspond with those in in vitro transport assay, suggesting that these methods might detect the different processes of drug-P-gp interaction. The combining of the ATPase activity assay and in vitro transport technologies provides us the insight into mechanisms of the membrane transport of drugs by P-gp.
\end{abstract}

Key words P-glycoprotein; ATPase activity; Sf9 insect cell; high throughput; multidrug resistance (MDR1); ABCB1

Human P-glycoprotein (P-gp/MDR1 or ABCB1) was identified because of its overexpression in cultured cancer cells associated with an acquired cross-resistance to multiple anticancer drugs. ${ }^{1-3)}$ While "P-glycoprotein" was initially thought to play a role in modulating cellular permeability (' $\mathrm{P}$ ' stands for permeability) to drugs, ${ }^{1)}$ it was later demonstrated to be an ATP-dependent efflux pump of hydrophobic anticancer drugs including colchitine, doxorubicin, daunorubicin, vincristine and VP16. ${ }^{4-6)}$ Historically, P-glycoprotein provided one of the mechanistic explanations for the multidrug resistance phenomenon. The function of P-gp as a mechanism of multidrug resistance has been extensively investigated.

Because of the great impact of P-gp on the pharmacokinetic profile of a variety of drugs, several in vitro experimental methods have been developed by pharmaceutical companies to screen P-gp substrates among newly synthesized compounds. In vitro transport study using Caco-2 monolayers is one of the most popular techniques to detect efflux activity of P-gp where basal-to-apical permeability of drugs was compared to apical-to-basal one. ${ }^{7-9)}$ Recently, MDR1transfected cell lines, MDR1-MDCK cells or LLCPK1MDR1 cells, have become available for the in vitro drug transport study. Since Caco-2 cells express the several efflux transporters, such as MRP2 or BCRP, MDR1-transfected cells are useful to identify the role of P-gp in drug efflux transport.

According to the rapid increase in the number of compounds to be analyzed for their possibility to interact with P-gp, the assay system having a higher throughput has been required in many pharmaceutical companies. Polli et al. have compared the potency to identify P-gp substrates and in- hibitors of Calcein-AM fluorescence assay, ATPase activity assay and cell monolayer transport assay, and summarized the advantage and disadvantage of each assay method. ${ }^{10)}$ Since Calcein-AM fluorescence assay detects the inhibition effect of test compounds to the Calcein AM transport by P-gp, this assay system cannot distinguish P-gp substrate from its inhibitor. ${ }^{11,12)}$ In contrast, in ATPase activity assay, transport activity of P-gp was determined by the amount of inorganic phosphate $(\mathrm{Pi})$ that was liberated according to the hydrolysis of ATP to ADP. This Pi liberation occurs in the transport process of substrates by P-gp. Therefore, this assay method is possible to distinguish the substrate from the inhibitor. ${ }^{13-16)}$

However, recent report has described the inconsistency between ATPase activity and transport rate of some substrates and inhibitors of P-gp. Drugs such as dexamethasone, daunorubicin and PSC833 are reported to be efficiently transported at the expense of only a low rate of ATP hydrolysis. ${ }^{10,17,18)}$ In order to identify the reason of this inconsistency, kinetic parameters of the interaction of P-gp and substrate drugs in both assay methods should be compared. In this study, therefore, we have developed a method to analyze the ATPase activity assay data according to Michaelis-Menten kinetics, then, the parameters were compared with those obtained in an in vitro drug transport study in MDR1-MDCKII cell monolayers.

\section{MATERIALS AND METHODS}

Materials For ATPase activity assay, glycine, ATP magnesium salt, Tris, Mes, EGTA, dithiothreitol, ouabain, and trichloroacetic acid were purchased from Nacalai tesque, Inc. 
(Kyoto, Japan). The human P-glycoprotein expressed in Sf9 cell membranes, as the positive control, was purchased from GENTEST/BD Biosciences (Woburn, MA, U.S.A.).

MDR1-MDCKII cells were obtained from The Netherlands Cancer Institute (Amsterdam, The Netherlands) and were used between passage numbers of 20 to 40 . Minimum Essential Medium Alpha Medium with L-glutamine, ribonucleosides and deoxyribonucleosides (MEMAM), nonessential amino acids (NEAA), fetal bovine serum (FBS), L-glutamine, trypsin $(0.25 \%)$-EDTA (1 mM), and antibiotic-antimycotic mixture $(10000 \mathrm{U} / \mathrm{ml}$ penicillin $\mathrm{G}, 10000 \mu \mathrm{g} / \mathrm{ml}$ streptomycin sulfate and $25 \mu \mathrm{g} / \mathrm{ml}$ amphotericin B in $0.85 \%$ saline) were purchased from Gibco Laboratories (Lenexa, KS, U.S.A.). Transwell (12 well/plate, $3.0-\mu \mathrm{m}$ pores, $0.9-\mathrm{cm}^{2}$ membrane surface area) was purchased from Nippon Becton Dickinson Company, Ltd. (Tokyo, Japan). Verapamil hydrochloride and quinidine sulfate were purchase from Nacalai Tesque, Inc., and vinblastine sulphate was purchased from Wako Pure Chemical Industries, Ltd. (Osaka, Japan). Verapamil hydrochloride [N-methyl $\left.{ }^{3} \mathrm{H}\right](85 \mathrm{Ci} / \mathrm{mmol})$ and quinidine $\left[9-{ }^{3} \mathrm{H}\right](20 \mathrm{Ci} / \mathrm{mmol})$ were purchased from American Radiolabeled Chemicals (St. Louis, MO, U.S.A.). All other compounds and reagents used were obtained from Nacalai tesque, Inc. or Wako Pure Chemical Industries, Ltd. or SIGMA Chemical Co.

MDR1-Overexpressing Sf9 Insect Cells To prepare MDR1-highly expressing cell lines, transduction of Sf9 insect cell line yielded a clone with substantial P-gp levels as described previously. ${ }^{15,16)}$ MDR1 was expressed in Spodoptera frugiperda Sf9 insect cells using the pFASTBAC1 vector, where the MDR1 cDNA was inserted between the $S a c I$ and XhoI restriction enzyme sites. Recombinant baculoviruses encoding the MDR1 cDNA was generated with the BAC-TO-BAC Baculovirus Expression Systems (Invitrogen) according to the manufacturer's instructions. Sf9 cells $\left(1 \times 10^{6} \mathrm{cell} / \mathrm{ml}\right)$ were then infected with the recombinant baculoviruses and cultured in EX-CELL ${ }^{\mathrm{TM}} 420$ Insect serum-free medium (JRH Bioscience, Levexa, KS, U.S.A.) at $26^{\circ} \mathrm{C}$ with gentle shaking. The expression of MDR1 in Sf9 cells increased during the incubation. Three to 4 days after incubation, cells were harvested by centrifugation. Cells were subsequently washed with phosphate buffer saline (PBS) at $4{ }^{\circ} \mathrm{C}$, collected by centrifugation, and stored at $-30^{\circ} \mathrm{C}$ until used.

The cell pellet was thawed quickly and diluted 40 -fold with a hypotonic buffer $(0.5 \mathrm{~mm}$ Tris/Hepes, $\mathrm{pH} 7.4$ and $0.1 \mathrm{~mm}$ EGTA) and then homogenized with a potter-Elvehjem homogenizer. After centrifugation at $9100 \times \mathbf{g}$, the supernatant was further centrifuged at $100000 \times \boldsymbol{g}$ for $30 \mathrm{~min}$. The resulting pellet was suspended in $250 \mathrm{~mm}$ sucrose containing $10 \mathrm{~mm}$ Tris/Hepes, $\mathrm{pH}$ 7.4. The crude membrane fraction was layered over $40 \%(\mathrm{w} / \mathrm{v})$ sucrose solution and centrifuged at $100000 \times \boldsymbol{g}$ for $30 \mathrm{~min}$. The turbid layer at the interface was collected, suspended in $250 \mathrm{~mm}$ sucrose containing $10 \mathrm{~mm}$ Tris/Hepes, $\mathrm{pH} 7.4$, and centrifuged at $100000 \times \boldsymbol{g}$ for $30 \mathrm{~min}$. The membrane fraction was collected and suspended in a small volume (150 to $250 \mu \mathrm{l})$ of $250 \mathrm{~mm}$ sucrose containing $10 \mathrm{~mm}$ Tris/Hepes, $\mathrm{pH}$ 7.4. After the measurement of protein concentration by the BCA Protein Assay Kit (PIERCE, Rockford, IL, U.S.A.), the membrane solution was stored at $-80^{\circ} \mathrm{C}$ until used.
ATPase Activity Assay System The ATPase activity of the isolated Sf9 cell membranes was determined by measuring Pi liberation ${ }^{19)}$ according to the procedure reported by Sarkadi et $a .^{20}{ }^{20}$ with some modifications. In the present study, we developed a high-speed screening protocol using 96-well plates. ${ }^{15)}$ Briefly, the Sf9 cell membranes $(2 \mu \mathrm{g}$ of protein per each well) were suspended in $10 \mu \mathrm{l}$ of the incubation medium containing $50 \mathrm{~mm}$ Tris-Mes (pH 6.8), $2 \mathrm{~mm}$ EGTA, $2 \mathrm{~mm}$ ouabain, $2 \mathrm{~mm}$ dithiothreitol, $50 \mathrm{~mm}$ potassium chloride, and $5 \mathrm{~mm}$ sodium azide. This medium was mixed with $10 \mu \mathrm{l}$ of a test compound solution and then pre-incubated at $37^{\circ} \mathrm{C}$ for $3 \mathrm{~min}$. The ATPase reaction was started by adding $20 \mu \mathrm{l}$ of $4 \mathrm{~mm}$ ATP solution to the reaction mixture, and the incubation was maintained at $37^{\circ} \mathrm{C}$ for $30 \mathrm{~min}$ or stopped immediately. After the incubation, the reaction mixture was mixed with $20 \mu \mathrm{l}$ of $5 \%$ trichloroacetic acid and subsequently with $42 \mu \mathrm{l}$ of "solution A" that contained $2 \mathrm{~N}$ $\mathrm{HCl}: 0.1 \mathrm{M} \mathrm{Na}_{2} \mathrm{MoO}_{4}=4: 3$, and $18 \mu \mathrm{l}$ of "solution B" that contained $0.084 \%(\mathrm{w} / \mathrm{v})$ malachaite green in $1 \%$ polyvinil alcohol solution. Thereafter, $120 \mu \mathrm{l}$ of "solution C" that contained $7.8 \%(\mathrm{w} / \mathrm{v}) \mathrm{H}_{2} \mathrm{SO}_{4}$ was added to the mixture. These mixing processes were automatically carried out in the HALCS-I system (BioTec Co., Ltd., Tokyo, Japan). The absorbance of each reaction mixture in the 96-well plates was photometrically measured at a wave length of $625 \mathrm{~nm}$ in Multiskan JX system (Dainippon Pharmaceuticals Co., Osaka, Japan). The amount of liberated phosphate was quantified based on the calibration line established with Pi standards $(0,2,6,11 \mathrm{nmol} /$ well $)$. In addition, each 96-well plate contained a positive control, in which Sf9 cell membranes were incubated with $10 \mu \mathrm{m}$ verapamil.

MDR1-MDCKII Cell Culture In present experiments, MDR1-MDCKII cells were cultured, passaged, and grown as described previously. ${ }^{21)}$ Cells were cultured at $37^{\circ} \mathrm{C}$ in a humidified air- $5 \% \mathrm{CO}_{2}$ atmosphere. For transport studies, cells were seeded onto Transwell filter membrane inserts at a density of $20 \times 10^{4}$ cells/insert. MEMAM supplemented with $10 \%$ FBS, $1 \%$ L-glutamine, 1\% NEAA, and 5\% antibioticantimycotic solution (basic culture medium, BCM) was used as seeding medium, and medium was replaced to fresh BCM after $3 \mathrm{~d}$. In addition, MDR1-MDCKII monolayers were ready for studies $5 \mathrm{~d}$ later.

Transepithelial electrical resistance (TEER) of MDR1MDCKII monolayers was measured routinely before and after the experiment with the Millicell(r)-ERS system (Millipore Corporation, Bedford, MA, U.S.A.). Before seeding cells, electrical resistances of the supporting filter and buffer medium were measured and subtracted from the total electrical resistance determined with the monolayer to calculate the TEER of the monolayer.

Transport Experiments Cell monolayers were pre-incubated in transport medium (TM, Hanks' Buffered Salt solution with $100 \mathrm{~mm}$ Hepes, $\mathrm{pH}$ 7.4) for $30 \mathrm{~min}$ at $37^{\circ} \mathrm{C}$. After preincubation, the study was initiated by adding the test drugs to the donor side and TM to the receiver side. Drug transport was observed at various concentrations $(0.1$ to $100 \mu \mathrm{M}$ ) in two directions (apical, AP to basal, BL and BL to AP). Samples were obtained from receiver side at 30, 60, 80, 100 and $120 \mathrm{~min}$. The permeability of drugs were measured under $\mathrm{pH}$ gradient conditions (apical $\mathrm{pH}=6.5$ and basal $\mathrm{pH}=7.4$ ). All experiments were performed at $37^{\circ} \mathrm{C}$. The 
Table 1. Effect of Quinidine on the Initial Absorbance (at Time 0) of Test Solution in Sf9 Control Cells

\begin{tabular}{|c|c|c|c|c|c|c|c|c|c|}
\hline Quinidine $(\mu \mathrm{M})$ & 0.0 & 0.5 & 1.0 & 2.0 & 5.0 & 10 & 20 & 50 & 100 \\
\hline Absorbance of test solution ${ }^{a)}$ & 0.764 & 0.762 & 0.728 & 0.663 & 0.621 & 0.495 & 0.379 & 0.240 & 0.170 \\
\hline S.E. $\left(\times 10^{-3}\right)$ & 6.26 & 5.36 & 5.25 & 7.74 & 7.47 & 4.33 & 6.02 & 10.1 & 5.92 \\
\hline Relative absorbance $(\%)^{b)}$ & 100 & 99.8 & 95.3 & 86.5 & 81.3 & 64.8 & 49.6 & 31.3 & 22.3 \\
\hline
\end{tabular}

Initial absorbance was measured at various quinidine-concentrations $(0.5-100 \mu \mathrm{M}) . a)$ Initial absorbance was measured at $625 \mathrm{~nm}$ in Sf9 control cells. $b$ ) Rate of detection of initial absorbance is expressed as a functional percentage of the absorbance without quinidine (concentration 0$)$ in Sf9 control cells. Data are represented as means \pm S.E. ( $n=4)$.

sample solution including radioisotope was incubated with $10 \mathrm{ml}$ of scintillation fluid for 6 to $12 \mathrm{~h}$ at room temperature, and the concentrations (total radioactivity) were determined with a liquid scintillation counter (LSC 3500, Aloka, Tokyo, Japan).

Data Analysis The amount of liberated phosphate $(P i(t), \mathrm{nmol} / \mathrm{min} / \mathrm{mg}$ protein) was calculated according to the following equation,

$$
P i(t)=\left(P i(t)_{\mathrm{MDR} 1}-P i(0)_{\mathrm{MDR} 1}\right)-\left(P i(t)_{\mathrm{control}}-P i(0)_{\mathrm{control}}\right)
$$

where $\operatorname{Pi}(t)_{\mathrm{MDR} 1}$ and $\operatorname{Pi}(0)_{\mathrm{MDR} 1}$ are the amount of liberated phosphate at time $t$ and 0 in MDR1-transduced Sf9 cell membranes, respectively and $P i(t)_{\text {control }}$ and $P i(0)_{\text {control }}$ are the amount of liberated phosphate at time $t$ and 0 in Sf9 control cell membranes, respectively.

The permeability $\left(P_{\text {eff }}, \mathrm{cm} / \mathrm{s}\right)$ of drugs was calculated according to the equation of

$$
P_{\text {eff }}=1 / A \cdot \operatorname{Co}(d Q / d t)
$$

where $A$ is membrane surface area, $C o$ is donor concentration at $t=0(\mu \mathrm{M})$, and $d Q / d t$ is amount of drug transported within a given time period.

\section{RESULTS}

ATPase Activity Detected by Inorganic Phosphate (Pi) Liberation ATPase activity was determined by the amount of Pi that was liberated according to the hydrolysis of ATP to ADP for energy consumption by P-gp. Shown in Fig. 1 is the relationship between the amount of liberated $\mathrm{Pi}$ and applied concentration of quinidine. At a lower concentration range, $\mathrm{Pi}$ liberation increased with the increase of the concentration of quinidine and reached a maximum value between 10 $20 \mu \mathrm{M}$, suggesting saturation of P-gp in this concentration range. However, Pi liberation decreased markedly at the higher concentration $(50,100 \mu \mathrm{M})$ of quinidine.

Since the test solution, ATPase reaction-mixture containing Sf9 cell membranes and ATP solution with/without test compound, contains a certain amount of $\mathrm{Pi}$ that is derived from ATP itself, in the ATPase activity assay, initial absorbance (at time 0 ) of the test solution is measured routinely in Sf9 control cells (P-gp not expressed) as a blank. Then the amount of liberated $\mathrm{Pi}$ is calculated from the absorbance after subtracting the blank value. In Table 1, the effect of quinidine on the initial absorbance of the test solution was observed. Although the same amount of Pi should be contained in all solutions, the initial absorbance of the test solution decreased with increasing quinidine concentration. At the highest concentration, $100 \mu \mathrm{M}$, the absorbance was only $22.3 \%$ of that without quinidine. This result suggests the possibility that quinidine added to the solution interferes the

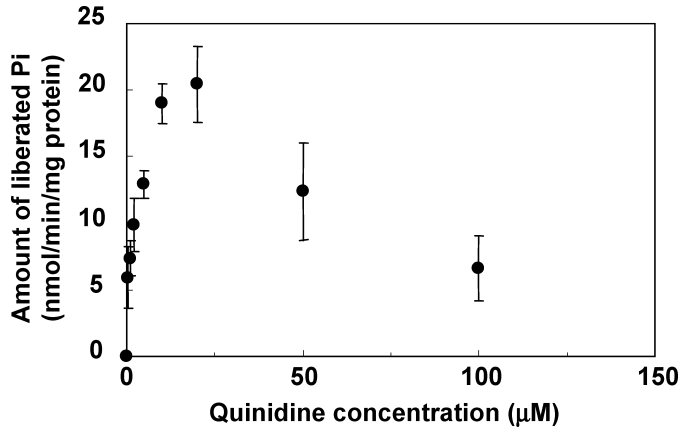

Fig. 1. Relationship between Amount of Liberated Pi and Applied Quinidine-Concentration in ATPase Activity Assay

The amount of liberated Pi was calculated according to the Eq. 1 in Data Analysis, Materials and Methods. Data are represented as means \pm S.E. $(n=4)$.

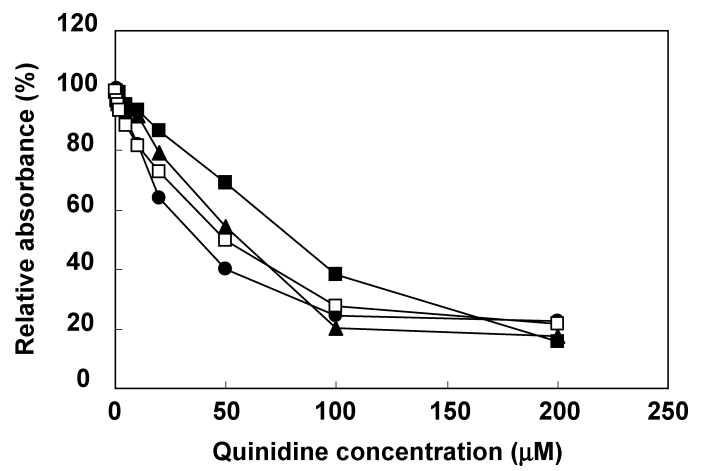

Fig. 2. Effect of Quinidine Concentration on the Photochemical Detection of Pi

Absorbance of Pi standard solution and ATP solution was measured in the presence of quinidine $(0.5-200 \mu \mathrm{M})$ and is expressed as the relative absorbance to that without quinidine. Twenty microliters of solution including $0.1 \mathrm{~mm}(\rightarrow), 0.2 \mathrm{~mm}(\rightarrow)$ and $0.3 \mathrm{~mm}(\leftarrow)$ of $\mathrm{Na}_{2} \mathrm{HPO}_{4}$ was added to the buffer solution to prepare Pi standard solution. ATP solution was prepared by adding $20 \mu \mathrm{l}$ of $4 \mathrm{~mm}$ ATP solution ( $\neg-)$. Each data point represents the mean of 3 experiments.

photometrical measurement of Pi.

The effect of quinidine on the detection of Pi was further investigated in Fig. 2, where the absorbance of the standard solution containing a defined amount of Pi (20 $\mu \mathrm{l}$ of $0.1,0.2$, $\left.0.3 \mathrm{~mm} \mathrm{Pi}\left(\mathrm{Na}_{2} \mathrm{HPO}_{4}\right)\right)$ was measured with various concentrations of quinidine. As a reference, initial absorbance of the test solution (containing $20 \mu \mathrm{l}$ of $4 \mathrm{~mm}$ ATP) was also depicted. In all solutions, relative absorbance (percentage to the absorbance without quinidine) decreased with increased quinidine concentration, indicating that quinidine interfered with the photometrical determination of Pi depending on its concentration. In Fig. 2, the pattern of decrease in absorbance was almost the same for all solutions. Therefore, it is reasonable to consider that the Pi liberation in Fig. 1 that was calculated from the absorbance of the test solution after 
ATPase reaction did not reflect the real activity of ATPase stimulated by P-gp.

Recalculation of the Amount of Pi Liberated in ATPase Activity Assay Assuming that the decrease in the absorbance in Fig. 2 is not dependent on the concentration of $\mathrm{Pi}$, but rather depends only on the concentration of quinidine, the real amount of Pi liberated in ATPase activity assay (Fig. 1) was recalculated. Absorbance of the test solution after ATPase reaction was corrected based on the percentage decrease in initial absorbance (at time 0 ) at each concentration of quinidine (Table 1), according to the following equation,

$$
A b s(t)_{\text {correction }}=A b s(t) \times \frac{A b s(0)_{\text {without quinidine }}}{A b s(0)_{\text {with quinidine }}}
$$

where $A b s(t)$ is the absorbance of the test solution after ATPase reaction and $A b s(t)_{\text {correction }}$ represents a corrected value of $A b s(t) . A b s(0)_{\text {with quinidine }}$ and $A b s(0)_{\text {without quinidine }}$ are initial absorbance (at time $=0$ ) of test solution with or without a defined concentration of quinidine, respectively.

As shown in Fig. 3, the amount of liberated Pi obtained

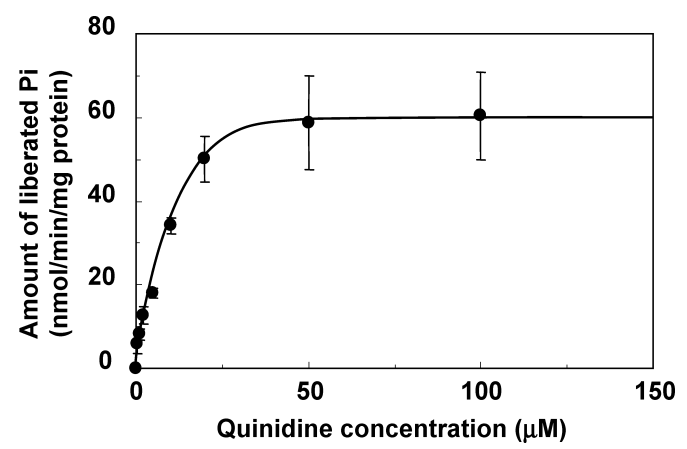

Fig. 3. Relationship between Amount of Liberated Pi and Applied Quinidine-Concentration in ATPase Activity Assay Calculated after Correction of Absorbance

The amount of liberated Pi was calculated according to the Eq. 1 in 'Data Analysis', Materials and Methods. Data are expressed as the mean \pm S.E. $(n=4)$.

(A)

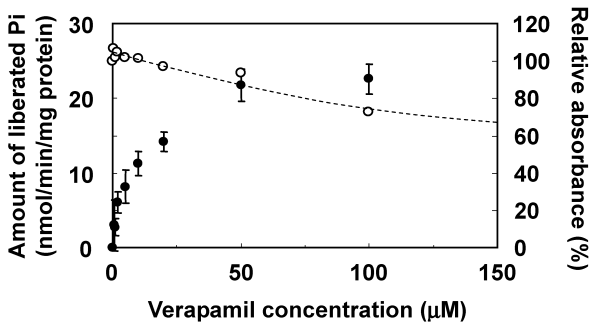

(C)

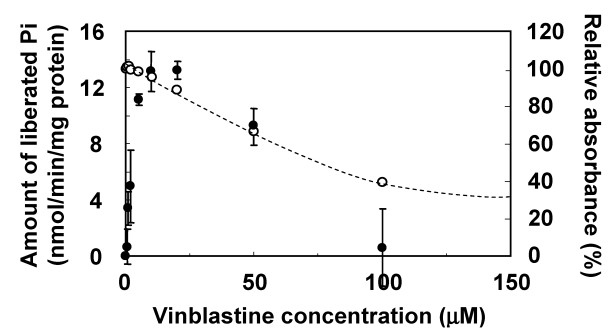

after correction of absorbance increased when quinidine was increased and reached a plateau level at a higher concentration range. In Fig. 3, Pi liberation was almost constant at 50 and $100 \mu \mathrm{M}$ of quinidine, indicating the possibility to fit to Michaelis-Menten kinetics.

The same experiments were carried out with verapamil and vinblastine as P-gp substrate drugs. As shown in Fig. 4, initial absorbance of the solution was also decreased by both drugs depending on their concentration. Although percentage decrease in absorbance was different among these three drugs, it is apparent that not only quinidine but also other Pgp substrate drugs such as verapamil and vinblastine interfere with the photochemical detection of $\mathrm{Pi}$.

The same method of correction on absorbance was applied to calculate the liberated amount of Pi in ATPase activity assay with verapamil and vinblastine (Fig. 5). In the case of vinblastine, Pi liberation calculated without correction (Fig. 5C) was markedly decreased at the high concentration range, while that calculated from the corrected absorbance reached

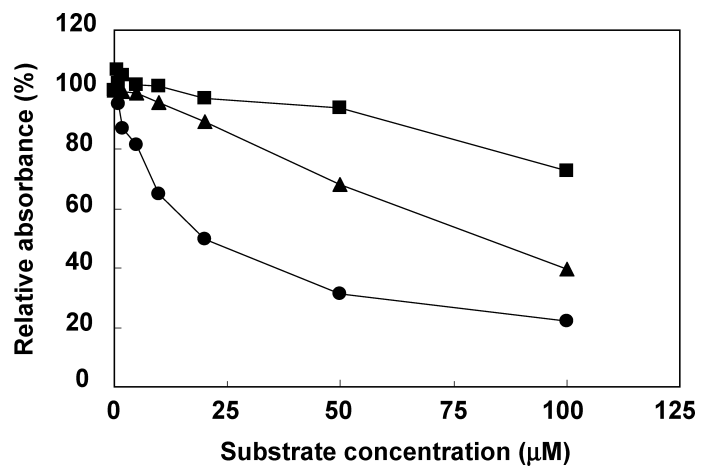

Fig. 4. Effect of Substrate Concentration on the Photochemical Detection of $\mathrm{Pi}$

Absorbance of ATP solution was measured in the presence of quinidine $(\rightarrow-)$, verapamil $(\rightarrow)$ and vinblastine $(\rightarrow)(0.5-100 \mu \mathrm{M})$ and are expressed as the relative absorbance to that without substrate. ATP solution was prepared by adding $20 \mu \mathrm{l}$ of $4 \mathrm{~mm}$ ATP solution. Each data point represents the mean of 3 experiments.

(B)

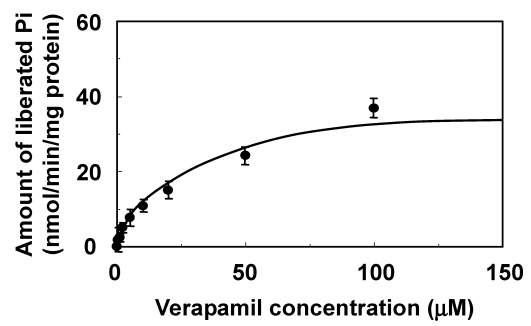

(D)

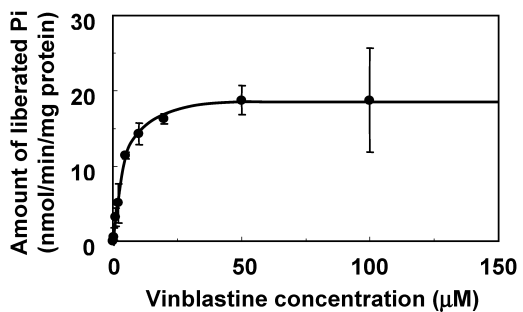

Fig. 5. Effect of Pi Absorbance Correction on the Relationship between Amount of Liberated Pi and Substrates-Concentration in ATPase Activity Assay

(A) Verapamil and (C) vinblastine relationship to the liberated amount of Pi calculated without correction and substrates concentration (solid symbols), and absorbance of ATP solution measured in the presence of substrate $(0.5-100 \mu \mathrm{M})$ are expressed as the relative absorbance to that without substrate (open symbol). (B) Verapamil and (D) vinblastine relationship between amount of liberated Pi and applied substrate-concentration in ATPase activity assay calculated after correction of absorbance. The amount of liberated Pi was calculated according to the Eq. 1 in 'Data Analysis', Materials and Methods. ATPase activity assay data and percentage data are represented as means \pm S.E. ( $n=4)$ and means $(n=3)$, respectively. 
Table 2. Kinetic Parameters of P-gp ATPase Activity Stimulated by Quinidine, Verapamil and Vinblastine in the Plasma Membrane Prepared from MDR1-Expressing Sf9 Insect Cells.

\begin{tabular}{cccc}
\hline \hline Substrate & $K_{\mathrm{m}}{ }^{a)}$ & $V_{\max }{ }^{b)}$ & $V_{\max } / K_{\mathrm{m}}{ }^{c)}$ \\
\hline Quinidine & 7.88 & 59.5 & 7.55 \\
Verapamil & 11.5 & 29.8 & 2.60 \\
Vinblastine & 5.76 & 21.3 & 3.70
\end{tabular}

Based on the Lineweaver-Burk plot of the corrected result in ATPase activity assay, $K_{\mathrm{m}}$ and $V_{\max }$ value were calculated. Units are $a$ ) $\left.\mu \mathrm{M} ; b\right) \mathrm{nmol} / \mathrm{min} / \mathrm{mg}$ protein; c) $\mathrm{ml} / \mathrm{min} / \mathrm{mg}$ protein

a plateau (Fig. 5D). The effect of verapamil on the photometrical detection of $\mathrm{Pi}$ was rather small compared to the other two drugs (Fig. 4); however, in Figs. 5A and B, the correction led to a significant change in the relationship between verapamil concentration and Pi liberation.

By applying the Michaelis-Menten equation to the relation between drug concentration and Pi liberation, affinity to P-gp $\left(K_{\mathrm{m}}\right)$ and the maximum amount of Pi liberation $\left(V_{\max }\right)$ were calculated for three drugs (Table 2). In all drugs, both $K_{\mathrm{m}}$ and $V_{\max }$ values could be calculated when corrected data of Pi liberation were used. The order of $K_{\mathrm{m}}$ value was verapamil $>$ quinidine $>$ vinblastine, indicating that verapamil has the lowest affinity to P-gp among three drugs. $V_{\max }$ value was highest for quinidine, and lowest for vinblastine.

In Vitro Transport Study in MDR1-MDCKII Monolayers In order to calculate the kinetic parameters on P-gpmediated drug efflux in the real transport process, absorptive (AP to BL) permeability of these P-gp substrate drugs were measured in MDR1-MDCKII cell monolayers. In Fig 6, AP to BL permeability of quinidine and verapamil at various donor (apical side) concentrations was illustrated. In this figure, the permeability of both drugs in the presence of potent P-gp inhibitor, alprenolol $(200 \mu \mathrm{M})$, was also depicted. For vinblastine, due to the cytotoxicity of the drug itself, transport study was not performed.

In both drugs, AP to BL permeability showed a sigmoidal relation to their donor concentration, and at the high concentration range, it reached a maximum value (Fig. 6). In the presence of alprenolol, the permeability of quinidine and verapamil at the low concentration range increased to the same level with the maximum permeability observed at the high concentration of drugs. These results suggested that P-gpmediated efflux was completely saturated at the high concentration range in both drugs and, therefore, the maximum permeability corresponds to the passive permeability of drugs in the monolayers.

Comparison of Parameters for P-gp-Mediated Transport A following model was applied to the relationship between the donor concentration of drugs $\left(C_{\mathrm{a}}\right)$ and their AP to BL flux $\left(J_{\mathrm{AB}}\right)$ across MDR1-MDCKII monolayers, assuming that $J_{\mathrm{AB}}$ can be expressed as the difference between passive $\left(J_{\text {passive }}\right)$ and P-gp-mediated flux $\left(J_{\text {P-gp }}\right)$.

$$
\begin{aligned}
J_{\mathrm{AB}} & =J_{\text {passive }}-J_{\mathrm{P}-\mathrm{gp}} \\
& =C L p_{\text {passive }} \cdot C_{\mathrm{a}}-J_{\max (\mathrm{pgp})} \cdot C_{\mathrm{a}} /\left(E C_{50}+C_{\mathrm{a}}\right)
\end{aligned}
$$

where $C L p_{\text {passive }}$ is passive clearance of the membrane (calculated as the passive permeability of the drug and the surface area of the membrane). In Eq. 4, P-gp-mediated efflux was defined by two parameters, $J_{\max (\mathrm{pgp})}$ and $E C_{50} . E C_{50}$ value rep-
(A)

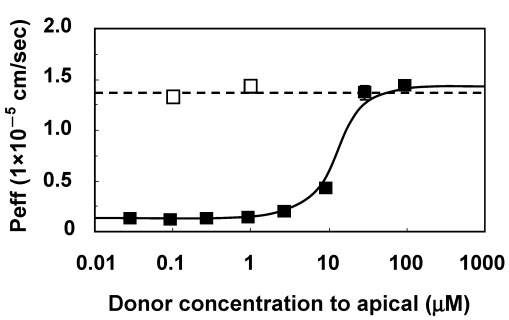

(B)

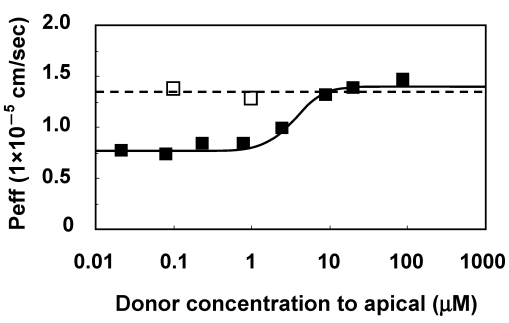

Fig. 6. Effect of Donor Concentration on Transcellular Transport of P-gp Substrates

MDR1-MDCKII monolayers were used for the transport experiments. Absorptive (AP to BL) permeability of (A) quinidine and (B) verapamil was measured at various donor concentrations $(0.03-100 \mu \mathrm{M})$. The experiment was performed with (--r-) or without $(\rightarrow-)$ P-gp inhibitor $(200 \mu \mathrm{m}$ alprenolol). Data are expressed as the mean \pm S.E. $(n=3)$.

Table 3. Kinetic Parameters of Transcellular Transport of Quinidine and Verapamil in MDR1-MDCKII Monolayer.

\begin{tabular}{cccc}
\hline \hline Substrate & $E C_{50}{ }^{a)}$ & $J_{\max (\mathrm{pgp})}{ }^{b)}$ & $\left.J_{\max (\mathrm{pgp})} / E C_{50}{ }^{c}\right)$ \\
\hline Quinidine & 16.4 & 13.3 & 0.808 \\
Verapamil & 2.85 & 1.26 & 0.442 \\
\hline
\end{tabular}

$E C_{50}$ and $J_{\max (\text { pgp })}$ values were obtained by fitting in vitro transport data to the Eq. 4 by the nonlinear least-squares method using MULTI program. Units are $a$ ) $\mu_{\mathrm{M}} ; b$ ) $\left.\mathrm{pmol} / \mathrm{min} / \mathrm{cm}^{2} ; c\right) \mu 1 / \mathrm{min} / \mathrm{cm}^{2}$.

resents the donor concentration of the drug at which P-gpmediated efflux became half of its maximum value $\left(J_{\max (\operatorname{pgp})}\right)$. Therefore, $E C_{50}$ could be regarded as an apparent affinity of drugs to P-gp.

In Table $3, E C_{50}$ and $J_{\max (\mathrm{pgp})}$ values were obtained for quinidine and verapamil by fitting in vitro data of transport study (Fig. 6) to the Eq. 4 by the nonlinear least-squares method using the MULTI program. ${ }^{22)} E C_{50}$ value of quinidine was 5.8 -fold larger than that of verapamil. This result was not consistent with the order of $K_{\mathrm{m}}$ in ATPase assay in Table 2, although $J_{\max (\mathrm{pgp})}$ value was larger for quinidine in both assay systems.

\section{DISCUSSION}

We have developed a high-throughput ATPase activity assay system and reported the usefulness of this system to characterize the substrate specificity of P-gp towards a variety of drugs. ${ }^{15,16)}$ However, recent report has described the inconsistency between ATPase activity and transport rate of some substrates and inhibitors of P-gp. Some drugs such as dexamethasone, doxorubicin and PSC 833 are reported to be efficiently transported at the expense of only a low rate of ATP hydrolysis. ${ }^{10,17,18)}$

In this study, quinidine and vinblastine showed a marked decrease in liberated $\mathrm{Pi}$ amount at a high-drug concentration 
after reaching a maximum value (Figs. 1, 5C). Although the maximum value of Pi liberation should indicate saturation of P-gp, these results suggested the inadequacy of applying the Michaelis-Menten equation directly to the data of ATPase activity assay. We have found that substrate drugs themselves interfered with the photometric detection of Pi (measurement of absorbance at $625 \mathrm{~nm}$ wave length) in a concentration-dependent manner. In the case of quinidine, the absorbance of the test solution detected with $100 \mu \mathrm{m}$ quinidine was only $23.3 \%$ of that without quinidine. Although the precise mechanisms underlying the interference of photometric detection of Pi by these drugs are not clear, we have suspected that this might be one of the reasons of inconsistent results between ATPase and transport assays of P-gp substrates. In order to obtain the kinetic parameters from ATPase assay data, therefore, we have tried to develop a method in which the net amount of Pi liberated can be calculated correctly.

Effect of Pi concentration on the interference of $\mathrm{Pi}$ absorbance by quinidine was demonstrated in Fig. 2. Although the result of $0.2 \mathrm{~mm} \mathrm{Pi}$ concentration deviated from those of other concentrations, it is reasonable to consider that the interference activity does not depend on Pi concentration. The reason why the significant deviation was observed only in $0.2 \mathrm{~mm} \mathrm{Pi}$ concentration is unclear, but plausibly, some experimental conditions (such as a temperature of the solution) might affect the interference activity by quinidine. Therefore, in order to correct $\mathrm{Pi}$ absorbance, the data of initial absorbance $(A b s(0)$ in Eq. 3) that was routinely measured as a blank $\left(P i(0)_{\text {control }}\right.$ in Eq. 1) in ATPase activity assay should be used.

Our method successfully improved the relation between Pi liberation and applied drug concentration of quinidine and vinblastine (Figs. 3, 5D). Corrected Pi liberation was constant at the high-drug concentration range after reaching a plateau, showing the possibility to be analyzed by MichaelisMenten equation to obtain the kinetic parameters, $K_{\mathrm{m}}$ and $V_{\max }$. In the case of verapamil, since absorbance-interference effect was rather small compared to other drugs, it was possible to calculate $K_{\mathrm{m}}$ and $V_{\max }$ from the data without correction (Fig. 5A) as $6.76 \mu \mathrm{M}$ and $22.03 \mathrm{nmol} / \mathrm{min} / \mathrm{mg}$ protein. The relationship between Pi liberation and verapamil concentration was significantly changed by the correction of absorbance, and both $K_{\mathrm{m}}$ and $V_{\max }$ values increased 1.7- and 1.4-folds, respectively.

Adachi et al. have also found that several P-gp substrates (such as quinidine, vinblastine, daunomycin, digoxin, loperamide and progesterone) interfered with the ATPase activity assay at the high concentration range. They have hypothesized that these compounds may interact with the membrane lipid and/or with a low-affinity inhibitory site of P-gp. ${ }^{18,23,24)}$ Although the substrate concentration with which Pi absorbance was apparently interfered was different from our results (e.g. for quinidine, $20 \mu \mathrm{M}$ in this study and $100 \mu \mathrm{M}$ in Adachi's report), their findings should be the same phenomenon with that observed in this study. The reason of inconsistency in the substrate concentration is not clear, but the delicate differences in the protocol of ATPase activity assay (such as the difference of the reagent for Pi detection) might affect the interference activity of substrate. Since some recent reports have also described the significant decrease in liberated $\mathrm{Pi}$ amount at a high concentration of $\mathrm{P}-\mathrm{gp}$ substrate ${ }^{17,18,25)}$ it would be possible to improve the quality of ATPase activity assay data by using the correction method proposed in this study. In our method, the data of initial absorbance $(A b s(0))$ that is routinely measured as a blank $\left(\operatorname{Pi}(0)_{\text {control }}\right)$ can be utilized and any additional measurements are not required.

Next, in order to ascertain the consistency in kinetic parameters of P-gp substrate interaction obtained in ATPase activity assay and in in vitro drug transport study, concentration dependency in the permeability of quinidine and verapamil was studied in MDR1-MDCKII cell monolayers (Fig. 6). As described by Eq. 4, AP to BL flux of drugs $\left(J_{\mathrm{AB}}\right)$ can be expressed as the difference between passive and P-gp-mediated efflux. Theoretically, the Michaelis-Menten equation can be applied to the P-gp-mediated efflux as

$$
\begin{aligned}
J_{\mathrm{P}-\mathrm{gp}} & =C L_{\mathrm{P}-\mathrm{gp}} \cdot C_{\text {bind }} \\
& =J_{\max (\mathrm{pgp})} \cdot C_{\text {bind }} /\left(K_{\mathrm{m}}+C_{\text {bind }}\right)
\end{aligned}
$$

where $C L_{\mathrm{P}-\mathrm{gp}}$ is $\mathrm{P}$-gp-mediated clearance of drugs and $C_{\mathrm{bind}}$ is the drug concentration at the vicinity of the drug binding-site of P-gp. However, because recent reports have revealed that the binding-site of P-gp to substrates might exist inside the lipid bilayer of cell membrane, ${ }^{26)}$ it is difficult (or almost impossible) to measure $C_{\text {bind }}$ experimentally. Therefore, in this report, we have used $E C_{50}$ instead of $K_{\mathrm{m}}$, which was defined by the drug concentration in the donor solution, to analyze the concentration-dependency of drug transport across MDR1-MDCKII monolayers. The $E C_{50}$ value in Eq. 4 could be regarded as an apparent affinity of drugs to P-gp and should be affected by the process of drug movement from the donor solution to the binding-site of P-gp.

Although $K_{\mathrm{m}}$ values obtained from ATPase activity assay were higher for verapamil than for quinidine, $E C_{50}$ value of verapamil obtained from in vitro transport study became lower than that of quinidine (Tables 2, 3). This inconsistency between ATPase activity and transport rate assays might reflect the different kinetics in each process in P-gp-mediated transport, the binding to P-gp, hydrolysis of ATP and transport across the membrane. ATPase assay is able to detect only first two processes, thus, in some substrates, ATPase assay might give the different results from those of transport assay.

In addition, it is possible to consider that the process of drug movement from the apical solution to the binding-site of P-gp might cause the inconsistency between $K_{\mathrm{m}}$ and $E C_{50}$ value. In Tables 2 and 3, a maximum P-gp transport activity $\left(J_{\max } / E C_{50}\right)$ and a maximum P-gp ATPase activity $\left(V_{\max } / K_{\mathrm{m}}\right)$ show the similar rank order for quinidine and verapamil. Both $J_{\max } / E C_{50}$ and $V_{\max } / K_{\mathrm{m}}$ are 2-3 times higher in quinidine than in verapamil. Because these values represent the transporter activity when the drug concentration is low enough compared to $K_{\mathrm{m}}$, the difference in $C_{\mathrm{a}}$ and $C_{\mathrm{bind}}$ in in vitro transport study can be cancelled, and then, a similar rank order might be obtained in both experiments.

Although the data of only three P-gp substrates presented here are not enough to generalize the usefulness of our newly developed method of correction of Pi liberation, it apparently improved the quality and enabled the kinetic analysis of ATPase activity assay data. By comparison of kinetic parameters obtained from ATPase activity and transport assays, the differences in both assay systems, and also the meanings of 
each parameter will be identified. Consequently, this new method brought a significant progress in ATPase assay not only as a high-throughput screening system, but also as a tool to investigate the mechanisms of drug interaction with P-gp.

Acknowledgements The experiments performed in Ishikawa's laboratory were supported, in part, by the NEDO International Joint Research Grant program "International standardization of functional analysis technology for genetic polymorphisms of drug transporters" and a research grant (No. 14370754) from the Japanese Society for the Promotion of Science.

\section{REFERENCES}

1) Juliano R. L., Ling V., Biochim. Biophys. Acta, 455, 152-162 (1976).

2) Ishikawa T., "Nature Publishing Group, 4, 154-160 (2003).

3) Borst P., Oude Elferink R., Ann. Rev. Biochem., 71, 537-592 (2002).

4) Thiebaut F., Tsuruo T., Hamada H., Gottesman M. M., Pastan I., Willingham M. C., Proc. Natl. Acad. Sci. U.S.A., 84, 7735-7738 (1987).

5) Hunter J., Hirst B. H., Adv. Drug Del. Rev., 25, 129-157 (1997).

6) Gao J., Murase O., Schowen R. L., Aube J., Borchardt R. T., Pharm. Res., 18, 171-176 (2001).

7) Kim R. B., Fromm M. F., Wandel C., Leake B., Wood A. J., Roden D. M., Wilkinson G. R., J. Clin. Invest., 101, 289-294 (1998).

8) Polli J. W., Jarrett J. L., Studenberg S. D., Humphreys J. E., Dennis S. W., Brouwer K. R., Woolley J. L., Pharm. Res., 16, 1206-1212 (1999).

9) Susanto M., Benet L. Z., Pharm. Res., 19, 457—462 (2002).
10) Polli J. W., Wring S. A., Humphreys J. E., Huang L., Morgan J. B., Webster L. O., Serabjit-Singh C. S., J. Pharmacol. Exp. Ther., 299, $620-628$ (2001).

11) Liminga G., Nygren P., Larsson R., Exp. Cell Res., 212, 291-296 (1994).

12) Tiberghien F., Loor F., Anticancer Drugs, 7, 568 - 578 (1996).

13) Scarborough G. A., J. Bioenerg. Biomembr., 27, 37-41 (1995).

14) Litman T., Nielsen D., Skovsgaard T., Zeuthen T., Stein W. D., Biochim. Biophys. Acta, 1361, 147-158 (1997).

15) Ishikawa T., Hirano H., Onishi Y., Sakurai A., Tarui S., Drug Metab. Pharmacokinet., 19, 1-14 (2004).

16) Ishikawa T., Sakurai A., Kanamori Y., Nagakura M., Hirano H., Takarada Y., Yamada K., Fukushima K., Kitajima M., Methods Enzymol., 400, 485-510 (2005).

17) Litman T., Zeuthen T., Skovsgaard T., Stein W. D., Biochim. Biophys. Acta, 1361, 159-168 (1997).

18) Adachi Y., Suzuki H., Sugiyama Y., Pharm. Res., 18, 1660-1668 (2001).

19) Carter S. G., Karl D. W., J. Biochem. Biophys. Methods, 7, 7-13 (1982).

20) Sarkadi B., Price E. M., Boucher R. C., Germann U. A., Scarborough G. A., J. Biol. Chem., 267, $4854-4858$ (1992).

21) Lentz K. A., Polli J. W., Wring S. A., Humphreys J. E., Polli J. E., Pharm. Res., 17, 1456-1460 (2000).

22) Yamaoka K., Tanigawara Y., Nakagawa T., Uno T., J. PharmacobioDyn., 4, 879-885 (1981).

23) Drori S., Eytan G. D., Assaraf Y. G., Eur. J. Biochem., 228, 1020-1029 (1995).

24) Borgnia M. J., Eytan G. D., Assaraf Y. G., J. Biol. Chem., 271, $3163-$ 3171 (1996).

25) Buxbaum E., Eur. J. Biochem., 265, 64-70 (1999)

26) Gottesman M. M., Pastan I., Annu. Rev. Biochem., 62, 385-427 (1993). 